Rev. Bras. Saúde Prod. Anim., Salvador, v.13, n.3, p.701-711 jul./set., 2012 http://www.rbspa.ufba.br ISSN 15199940

\title{
Perfil de sensibilidade antimicrobiana in vitro de Staphylococcus aureus isolado de mastite subclínica bovina
}

\author{
"In vitro" antimicrobial susceptibility of "Staphylococcus aureus" isolated from bovine \\ subclinical mastitis
}

\begin{abstract}
SILVA, Elizabete Rodrigues da ${ }^{1 *}$; PEREIRA, Angélica Martina Gonçalves ${ }^{1}$; MORAES, Wisley da Silva ${ }^{1}$; SANTORO, Kleber Régis ${ }^{1}$; SILVA, Tatiana Rossana Móta ${ }^{1}$
\end{abstract}

\author{
${ }^{1}$ Universidade Federal Rural de Pernambuco, Unidade Acadêmica de Garanhuns, Garanhuns, \\ Pernambuco, Brasil. \\ *Endereço para correspondência: elizabete@uag.ufrpe.br
}

\section{RESUMO}

Com o objetivo de determinar o perfil de sensibilidade antimicrobiana in vitro foram estudadas 83 amostras de Staphylococcus aureus isoladas de mastite subclínica bovina, em rebanhos leiteiros do município de Garanhuns, PE. Ao se utilizar a técnica de difusão em ágar, 79 (95\%), amostras demonstraram resistência à penicilina $\mathrm{G}$ e 73 $(88 \%)$ à ampicilina. Frente às demais drogas testadas, $100 \%$ das amostras foram sensíveis à cefalotina, 79 (95\%) à enrofloxacina, 77 (93\%) à tetraciclina, $76(92 \%)$ à eritromicina e clindamicina, e $65(78 \%)$ à gentamicina. Das amostras provenientes de animais com infecção intramamária recente, 91 e $87 \%$ foram resistentes à penicilina e ampicilina, enquanto 100 e $89 \%$ daquelas de infecção crônica foram resistentes àquelas drogas, nessa ordem. Dentro da categoria "infecção crônica", houve diferença significativa entre a eficácia de penicilina e ampicilina $(\mathrm{P}<0,01)$. A resistência a dois antimicrobianos simultaneamente foi a mais frequente, cujo perfil penicilina+ampicilina observado em 55 (66\%) das amostras avaliadas. Os resultados deste trabalho demonstraram que a resistência às penicilinas é frequente em Staphylococcus aureus isolado de casos de mastite subclínica em rebanhos de bovinos leiteiros do município de Garanhuns, Pernambuco.

Palavras-chave: mastite bovina, penicilinas, resistência antimicrobiana.

\section{SUMMARY}

Eighty-three strains of Staphylococcus aureus isolated from bovine subclinical mastitis were studied in order to evaluate the in vitro antimicrobial sensibility. Using agar diffusion technique, $79(95 \%)$ strains were resistant to penicillin $\mathrm{G}$ and 73 (88\%) to ampicillin. Against for the others tested drugs $83(100 \%)$ were sensitive to cephalothin, 79 (95\%) to enrofloxacin, $77(93 \%)$ to tetracycline, 76 (92\%) to erythromycin and clindamycin, and 65 (78\%) to gentamicin. samples From those animals with recent intramammary infection, 91 and $87 \%$ strains were resistant to penicillin and ampicillin, while from chronic infection 100 and $89 \%$ were resistant to those drugs, respectively. There was significant difference among the effectiveness of penicillin and ampicillin $(\mathrm{P}<0.01)$ for chronic infection category. Resistance to two antibiotics simultaneously was the most frequent with the profile penicillin+ampicillin observed in $55(66 \%)$ of the studied strains. The results showed that resistance to penicillins frequently occurs in Staphylococcus aureus isolated from subclinical mastitis in dairy cattle herds in the municipality of Garanhuns, PE, Brazil.

Keywords: antibiotic resistance, bovine mastitis, penicillins. 


\section{INTRODUÇÃOO}

Embora uma variedade de agentes microbianos esteja envolvida com a etiologia da mastite bovina, Staphylococcus aureus (S. aureus) é o agente infeccioso mais prevalente de infecção intramamária (IMI) e está relacionado a mais de $80 \%$ dos casos (PELLEGRINO et al., 2011). Em vacas infectadas por essa bactéria, estima-se que a produção de leite seja reduzida em até $15 \%$, enquanto um único quarto mamário infectado poderá ter a produção reduzida em até $45 \%$ (BRITO \& BRITO, 1998).

Estudos realizados no Brasil, indicam que $S$. aureus está amplamente distribuído nos rebanhos leiteiros nacionais, com frequências que variam de 32,9 a $70,9 \%$ (LARANJA \& MACHADO, 1994; MOREIRA et al., 1997; ANDRADE et al., 2000; FREITAS et al., 2005; ZANETTE et al., 2010).

A alta prevalência de $S$. aureus está relacionada à habilidade desse patógeno de invadir e se estabelecer profundamente nos tecidos da glândula mamária, o que torna a mastite por esse agente um desafio aos programas de controle e prevenção de IMI. Além disso, a capacidade de desenvolver resistência aos antimicrobianos também contribui para a alta prevalência dessa espécie bacteriana (BRITO \& BRITO, 1998; SABOUR et al., 2004; MORONI et al., 2006; ZAFALON et al., 2008).

A terapia antimicrobiana é um componente importante de programas de controle e prevenção das mastites e é determinante para a eliminação da doença em rebanho leiteiro (PELLEGRINO et al., 2011). Com os $\beta$-lactâmicos, representa o principal grupo de drogas utilizado para IMI, causada por Grampositivos, tanto durante a lactação quanto à secagem do animal (BETANCOURT et al., 2003; ZSCHOCK et al., 2011).

Resultados de trabalhos realizados no Brasil e, particularmente, no Estado de Pernambuco, demonstram que a eficácia de alguns $\beta$-lactâmicos frente a isolados de mastite bovina é bastante limitada (MOREIRA et al., 1997; BUENO et al., 2003; FREITAS et al., 2005; MEDEIROS et al., 2009; FERREIRA et al., 2010; ZANETTE et al., 2010).

Semelhante ao que ocorre com os $\beta$ lactâmicos, o uso abusivo de outras classes de drogas, seja para o controle de mastites, seja para o de outras doenças infecciosas, também contribui para o aumento da taxa de resistência antimicrobiana em diversos patógenos (BARKEMA et al. 2006; AMÁBILECUEVAS, 2010).

Dessa forma, objetivou-se, neste trabalho, determinar o perfil de sensibilidade antimicrobiana in vitro, de amostras de Staphylococcus aureus isoladas de mastite subclínica em rebanhos de bovinos leiteiros do município de Garanhuns, Pernambuco.

\section{MATERIAL E MÉTODOS}

As amostras de $S$. aureus, avaliadas neste estudo, foram isoladas de casos de mastite subclínica de vacas pertencentes a três rebanhos leiteiros comerciais, localizados em Garanhuns, município pertencente à região do Agreste Meridional pernambucano. Os animais foram amostrados durante os meses de abril a agosto de 2010, a intervalos não superiores a 30 dias. Tanto a colheita do leite, quanto a lactocultura, foram realizadas de acordo com as recomendações do National Mastitis Council - NMC (2004). Amostras, cujo cultivo primário demonstrou crescimento 
de três ou mais tipos bacterianos, foram consideradas contaminadas e descartadas. Quando colônias sugestivas de S. aureus foram observadas, juntamente com as contaminantes, considerou-se a amostra positiva para esse patógeno. Considerouse infecção intramamária recente quando esse agente foi isolado em uma única amostra de leite, ao longo do período amostrado, e infecção crônica quando $S$. aureus foi isolado em duas ou mais amostras consecutivas de leite ou em duas de uma série de três amostras consecutivas (DOHOO et al., 2011; PERSSON et al., 2011). Durante o estudo não foram isolados $S$. aureus de casos de mastite clínica.

Para a identificação de $S$. aureus, utilizou-se a chave de identificação de Staphylococci sugerida por Kloos (1990). Todos os testes foram realizados de acordo com Quinn et al. (1994) e MacFaddin (2000). As amostras foram mantidas congeladas em meio composto de $15 \%$ de glicerol (v/v), até a realização dos testes de susceptibilidade antimicrobiana.

A sensibilidade aos antimicrobianos foi determinada pelo método de difusão em placa, segundo a recomendação do CLSI (ANON, 2008). Os critérios de escolha de drogas sugeridos pelo CLSI também foram considerados. Dessa forma, os seguintes grupos/drogas foram selecionados: Penicilinas naturais/Penicilina G 10 U.I.; Aminopenicilinas/Ampicilina 10mcg; Penicilinas Resistentes às Penicilinases (PRPs)/ Oxacilina 1mcg; Cafalosporinas/Cefalotina 30 $3 \mathrm{mcg}$, Quinolonas/Enrofloxacina $5 \mathrm{mcg}$, Aminoglicosídeos/Gentamicina $10 \mathrm{mcg}$, Tetraciclinas/Tetraciclina 30mcg, Macrolídeos/Eritromicina $15 \mathrm{mcg}$ e Lincosamidas/Clindamicina $2 \mathrm{mcg}$.

As drogas utilizadas representaram grupos indicados a animais de produção e compõem formulações para o tratamento de mastites e de infecções localizadas em outros sistemas. As zonas de inibição do crescimento microbiano foram interpretadas de acordo com os limites estabelecidos pelo CLSI (ANON, 2008). Para o controle de qualidade dos testes e reprodutibilidade dos resultados, utilizou-se a cepa ATCC 25923.

Foram estabelecidas as frequências absoluta e relativa para cada droga testada. Para comparar a resposta das amostras das categorias "infecção recente" e "crônica" frente a cada antimicrobiano, realizou-se a análise de variância não paramétrica com oemprego do teste de Kruskall-Wallis. Quando ocorreram diferenças significativas, realizou-se o teste LSD de Fisher a fim de verificar a direção das diferenças. Em ambos os testes, o nível de probabilidade foi de $1 \%$. Para as análises utilizou-se o software SAS for Windows, $\quad$ versão $\quad 9.2 ® \quad$ (SAS INSTITUTE, ).

\section{RESULTADOS E DISCUSSÃO}

O perfil de sensibilidade das 83 amostras de $S$. aureus avaliadas neste estudo é demonstrado na Tabela 1. As taxas de resistência observadas para penicilina $G$ e ampicilina indicam que, nos rebanhos estudados, $S$. aureus foi submetido à uma alta pressão seletiva frente àquelas drogas. Alguns investigadores, ao trabalharem com amostras de Staphylococci isoladas em rebanhos localizados em outros municípios do Agreste pernambucano, também observaram altas taxas de resistência àquelas penicilinas. Freitas et al. (2005), aoavaliarem amostras de S. aureus e outros Staphylococcus coagulase positiva, relataram $80 \%$ de resistência para a penicilina $\mathrm{G}$, enquanto 
Medeiros et al. (2009) relataram 72,8\% de resistência para a ampicilina. Os resultados do presente estudo, juntamente com os desses investigadores, indicam que as taxas de resistência na região encontram-se em crescimento.

Taxas elevadas de resistência às penicilinas também foram observadas em outros estados brasileiros. Relatos referem que Staphylococci isolados de mastite bovina, com fenótipo de resistência às penicilinas, estão presentes nos rebanhos nacionais em uma frequência que varia de 10 a $57 \%$ para oxacilina, 46 a $100 \%$ para penicilina e 78,9 a $100 \%$ para ampicilina (MOREIRA et al., 1997; ANDRADE et al., 2000; BUENO et al., 2003; CORREAA et al., 2005; FERREIRA et al., 2010; ZANETTE et al., 2010).

Tabela 1. Perfil de sensibilidade antimicrobiana de amostras de Staphylococcus aureus isoladas de mastite bovina, Garanhuns, PE, 2011

\begin{tabular}{lrrrrcc}
\hline & \multicolumn{9}{c}{ Perfil } \\
\hline Antimicrobiano & \multicolumn{2}{c}{ Sensível } & \multicolumn{2}{c}{ Intermediário } & \multicolumn{2}{c}{ Resistente } \\
\cline { 2 - 8 } & $\mathrm{n}$ & $\%$ & $\mathrm{n}$ & $\%$ & $\mathrm{n}$ & $\%$ \\
\hline Ampicilina & 10 & 12 & - & - & 73 & 88 \\
Cefalotina & 83 & 100 & - & - & - & - \\
Clindamicina & 76 & 92 & 2 & 2 & 5 & 6 \\
Enrofloxacina & 79 & 95 & 4 & 5 & - & - \\
Eritromicina & 76 & 92 & 4 & 5 & 3 & 4 \\
Gentamicina & 65 & 78 & 3 & 4 & 15 & 18 \\
Oxacilina & 82 & 99 & 1 & 1 & - & - \\
Penicilina & 4 & 5 & - & - & 79 & 95 \\
Tetraciclina & 77 & 93 & 1 & 1 & 5 & 6 \\
\hline
\end{tabular}

Entre países, a taxa de $S$. aureus resistente às penicilinas é bastante variável. Na Coreia do Sul, Nam et al. (2011) relataram taxa superior a $66 \%$ e aumento da resistência naquele país. Reinoso et al. (2007), na Argentina, e Sahebekhtiari et al. (2011), no Irã, relataram taxas superiores a 55 e de $28 \%$, respectivamente. As diferenças observadas entre países podem estar associadas às diferentes metodologias utilizadas pelos autores, como também, às diferentes políticas de controle de antimicrobianos. No Brasil, por exemplo, não existe controle da venda e uso de antimicrobianos de uso veterinário, o que permite a livre comercialização dessas drogas, inclusive sem prescrição de médico veterinário. Isso contribui, seguramente, para o surgimento de resistências, devido à escolha inadequada da droga, via de administração, dosagem e duração do tratamento.

A resistência à drogas do grupo das penicilinas em $S$. aureus isolados de IMI é uma preocupação mundial. Os motivos são vários, no entanto, por ora destacam-se: o potencial risco da transmissão de resistência para bactérias causadoras de doenças em humanos, de modo a restringir ou impossibilitar o seu uso em casos graves (BETANCOURT et al., 2003); e a baixa probabilidade de cura microbiológica das mastites por 
aquele agente portador de tal fenótipo (BARKEMA et al., 2006).

A baixa probabilidade de cura das mastites por $S$. aureus resistentes às penicilinas não é restrita apenas a esses e outros $\beta$-lactâmicos, mas, também, à drogas não $\beta$-lactâmicas (BARKEMA et al., 2006). De acordo com esses autores, essa característica ocorre devido à proximidade no genoma de $S$. aureus de genes que codificam para mecanismos de resistência e para fatores de virulência, o que permite a coexpressão dos mesmos e o escape da bactéria da ação de grupos de antimicrobianos distintos. Dessa forma, para Barkema e coautores, animais que portem $S$. aureus, com aquele perfil, não devem receber tratamento antimicrobiano.

A baixa taxa de cura de mastites, causadas por cepas de $S$. aureus resistentes às penicilinas, foi observada tanto em esquemas de terapia intramamária, quanto em esquemas de terapia combinada - intramamária + sistêmica (TAPONEN et al., 2003) -. Para esses autores, essa característica sugere uma maior virulência de amostras "penicilina resistente" ou uma baixa eficácia das drogas, devido a fatores relacionados à farmacodinâmica e farmacocinética das mesmas na glândula mamária.

A via de administração também implica no surgimento de resistência. De acordo com Hillerton \& Kliem (2002), o risco de selecionar cepas resistentes é muito maior, ao se utilizar a via parenteral do que a intramamária, uma vez que a quantidade da droga utilizada em tratamentos sistêmicos é muito superior àquela usada nos tratamentos locais. Durante a realização deste trabalho, observou-se, nos rebanhos amostrados, uso frequente de antimicrobianos por via parenteral e, em alguns rebanhos, era a preferencial para o tratamento de mastite e de outras infecções.
Neste estudo, 99\% dos isolados de $S$. aureus foram sensíveis a oxacilina. Esse antimicrobiano é indicado para pesquisar a resistência às PRPs, inclusive à meticilina, uma vez que apresenta maior estabilidade nos testes de susceptibilidade in vitro (ANON, 2004). Em amostras isoladas, em distintas regiões do estado pernambucano, outros investigadores relataram resistência àquele grupo de penicilina, com taxas que variavam de 11,3 a $75 \%$ para a amoxicilina e $15 \%$ para a oxacilina (FREITAS et al., 2005; MEDEIROS et al., 2009). Os resultados do presente trabalho sugerem que PRPs ainda são uma opção para o tratamento das mastites nos rebanhos amostrados.

$O$ perfil de sensibilidade, sensibilidade intermediária e resistência das amostras por categoria (infecção recente $\mathrm{e}$ infecção crônica) pode ser visualizado na Tabela 2. Em ambas as categorias, mais de $89 \%$ das amostras foram sensíveis à maioria das drogas avaliadas.

Na tabela 3, estão sistematizados os resultados das análises realizadas para cada antimicrobiano, por categoria de infecção. A análise de variância pelo teste de Kruskall-Wallis indicou diferenças significativas para a resposta das amostras de $S$. aureus frente às diferentes drogas testadas $(\mathrm{P}<0,01)$. Em ambas as categorias, as maiores taxas de resistência foram observadas para a penicilina, seguidos por ampicilina e gentamicina, nessa ordem, e as maiores taxas de sensibilidade para a cefalotina e oxacilina. A comparação pelo teste LSD demonstrou que, dentro da categoria infecção recente, penicilina e ampicilina não diferiram entre si $(\mathrm{P}>0,01)$, enquanto que, na categoria infecção crônica, houve diferença significativa entre estas duas drogas, com a maior taxa de resistência apresentada frente à penicilina $(\mathrm{P}<0,01)$. 
Rev. Bras. Saúde Prod. Anim., Salvador, v.13, n.3, p.701-711 jul./set., 2012 http://www.rbspa.ufba.br ISSN 15199940

Tabela 2. Perfil de sensibilidade antimicrobiana de Staphylococcus aureus isolado de infecção intramamária bovina "recente" e "crônica", Garanhuns, PE, 2011

\begin{tabular}{lcccccccccccccc}
\hline & \multicolumn{1}{c}{ Infecção recente } & \multicolumn{1}{c}{ Infecção crônica } \\
\hline \multirow{2}{*}{ Antimicrobiano } & \multicolumn{1}{c}{$\mathrm{S}^{*}$} & \multicolumn{1}{c}{$\mathrm{I}^{*}$} & \multicolumn{1}{c}{$\mathrm{R}^{*}$} & \multicolumn{3}{c}{$\mathrm{S}^{*}$} & \multicolumn{1}{c}{$\mathrm{I}^{*}$} & \multicolumn{1}{c}{$\mathrm{R}^{*}$} \\
\cline { 2 - 16 } & $\mathrm{n}$ & $\%$ & $\mathrm{n}$ & $\%$ & $\mathrm{n}$ & $\%$ & $\mathrm{~N}$ & $\%$ & $\mathrm{n}$ & $\%$ & $\mathrm{n}$ & $\%$ \\
\hline Ampicilina & 6 & 13 & - & - & 40 & 87 & 4 & 11 & - & - & 33 & 89 \\
Cefalotina & 46 & 100 & - & - & - & - & 37 & 100 & - & - & - & - \\
Clindamicina & 42 & 91 & 1 & 2 & 3 & 7 & 34 & 92 & 1 & 3 & 2 & 5 \\
Enrofloxacina & 44 & 96 & 2 & 4 & - & - & 35 & 95 & 2 & 5 & - & - \\
Eritromicina & 43 & 93 & 1 & 2 & 2 & 4 & 33 & 89 & 3 & 8 & 1 & 3 \\
Gentamicina & 35 & 76 & 3 & 7 & 8 & 17 & 30 & 81 & - & - & 7 & 19 \\
Oxacilina & 45 & 98 & 1 & 2 & - & - & 37 & 100 & - & - & - & - \\
Penicilina & 4 & 9 & - & - & 42 & 91 & - & 0 & - & - & 37 & 100 \\
Tetraciclina & 42 & 91 & 1 & 2 & 3 & 7 & 35 & 95 & - & - & 2 & 5 \\
\hline
\end{tabular}

${ }^{*} \mathrm{~S}=$ sensível; $\mathrm{I}=$ intermediário; $\mathrm{R}=$ resistente

Tabela 3. Escores dos antimicrobianos testados contra Staphylococcus aureus em função da categoria de infecção, Garanhuns, 2011

\begin{tabular}{lcc}
\hline \multirow{2}{*}{ Antimicrobiano } & \multicolumn{2}{c}{ Infecção } \\
\cline { 2 - 3 } & Recente & Crônica \\
\hline Ampicilina & $4,04^{\mathrm{c}}$ & $2,71^{\mathrm{c}}$ \\
Cefalotina & $31,01^{\mathrm{a}}$ & $25,06^{\mathrm{a}}$ \\
Clindamicina & $28,31^{\mathrm{a}}$ & $23,03^{\mathrm{a}}$ \\
Enrofloxacina & $29,66^{\mathrm{a}}$ & $22,35^{\mathrm{a}}$ \\
Eritromicina & $28,99^{\mathrm{a}}$ & $23,71^{\mathrm{b}}$ \\
Gentamicina & $23,59^{\mathrm{b}}$ & $20,32^{\mathrm{b}}$ \\
Oxacilina & $33,34^{\mathrm{a}}$ & $25,06^{\mathrm{a}}$ \\
Penicilina & $2,69^{\mathrm{c}}$ & $0,00^{\mathrm{d}}$ \\
Tetraciclina & $28,31^{\mathrm{a}}$ & $23,71^{\mathrm{a}}$ \\
\hline
\end{tabular}

Médias seguidas da mesma letra na mesma coluna, não diferem entre si pelo teste LSD de Fisher ao nível de $1 \%$ de probabilidade.

Os resultados permitem sugerir que, nos rebanhos avaliados, os animais cronicamente infectados são reservatórios de $S$. aureus penicilinas resistente e disseminam tal fenótipo. Por sua vez, essa disseminação está relacionada à baixa taxa de cura microbiológica das mastites por $S$. aureus resistentes às penicilinas, como observado por Taponen et al. (2003) e Barkema et al. (2006), de modo a permitir que animais, cronicamente infectados, permaneçam no rebanho e disseminem cepas resistentes para outros animais.

Na Tabela 4, demonstram-se as taxas de multirresistência das amostras avaliadas. Do total, 78 (94\%) amostras foram resistentes a duas ou mais drogas, simultaneamente. A resistência a duas drogas foi a mais frequente, cujo perfil penicilina+ampicilina 
Rev. Bras. Saúde Prod. Anim., Salvador, v.13, n.3, p. 701-711 jul./set., 2012 http://www.rbspa.ufba.br ISSN 15199940

(PEN+AMP), observado em $55(66 \%)$ amostras. Para as categorias infecção recente e crônica, a taxa de multirresistência foi de 92 e $97 \%$, nessa ordem.

Tabela 4. Multirresistência em amostras de Staphylococcus aureus isoladas de infecção intramamária bovina, Garanhuns, PE, 2011

\begin{tabular}{|c|c|c|c|c|c|c|c|c|c|c|c|}
\hline \multirow{3}{*}{ Categoria } & \multicolumn{11}{|c|}{ Quantidade de antimicrobiano } \\
\hline & \multicolumn{2}{|c|}{$0 *$} & \multicolumn{2}{|c|}{1} & \multicolumn{2}{|c|}{2} & \multicolumn{2}{|c|}{3} & \multicolumn{2}{|c|}{4} & \multirow{2}{*}{ Tota } \\
\hline & $\mathrm{n}$ & $\%$ & $\mathrm{n}$ & $\%$ & $\mathrm{n}$ & $\%$ & $\mathrm{n}$ & $\%$ & $\mathrm{n}$ & $\%$ & \\
\hline Infecção recente & 1 & 2 & 3 & 7 & 32 & 70 & 10 & 22 & - & - & 46 \\
\hline Infecção crônica & - & - & 1 & 3 & 28 & 76 & 6 & 16 & 2 & 5 & 37 \\
\hline Total & 1 & 1 & 4 & 5 & 60 & 72 & 16 & 19 & 2 & 2 & 83 \\
\hline
\end{tabular}

*Amostra sensível a todos os antimicrobianos testados.

Taxas elevadas de multirresistência para isolados de S. aureus e Staphylococcus coagulase positiva de rebanhos da região do Agreste de Pernambuco também foram relatadas por Freitas et al. (2005) e Medeiros et al. (2009) as quais variavam de 69 a $100 \%$ das amostras, respectivamente. A taxa de multirresistência observada neste e em outros trabalhos é preocupante, visto que amostras com esse perfil serão refratárias a maioria dos esquemas terapêuticos disponíveis para o tratamento das mastites em animais de produção.

A alta taxa de resistência simultânea a penicilina e ampicilina, como observado neste trabalho, sugere que o mesmo mecanismo de resistência seja responsável pela inativação de ambas as drogas. Além disso, se considerarmos que $S$. aureus, cujo fenótipo PEN+AMP resistente responde a vários grupos de drogas $\beta$-lactâmicas e não $\beta$-lactâmicas (BARKEMA et al., 2006), os resultados do presente estudo sugerem que tal fenótipo deve ser utilizado para guiar a seleção de esquema terapêutico mais adequado no rebanho no qual foi detectado.

Com relação às demais drogas testadas (e como demonstrado nas Tabelas $1 \mathrm{e}$ 2), $100 \%$ das amostras foram sensíveis à cefalotina, 79 (95\%) à enrofloxacina, $77(93 \%)$ à tetraciclina, $76(92 \%)$ à eritromicina e clindamicina, e $65(78 \%)$ à gentamicina.

A taxa de sensibilidade dos isolados de $S$. aureus avaliados frente à cefalotina deve ser interpretada com cautela, visto tratar-se de um $\beta$-lactâmico. Além disso, tal resultado não deve ser extrapolado para as demais cefalosporinas por apresentarem diferenças quanto ao espectro e resistência às $\beta$-lactamases (ANON, 2008). Por outro lado, a total eficácia da cefalotina in vitro é sugestiva da produção de penicilinases pelos isolados de $S$. aureus avaliados.

Embora tetraciclina e enrofloxacina sejam usadas frequentemente em animais de produção para o tratamento de diversas infecções, os resultados do presente trabalho indicam que essas drogas são pouco utilizadas nos rebanhos amostrados. Para a gentamicina, os resultados sugerem que o uso dessa ou de outras drogas do grupo deve ser feito com critérios de forma a não favorecer o aumento da resistência a essas substâncias.

Os resultados observados para eritromicina e clindamicina sugerem que os macrolídeos e lincosamidas podem ser uma opção terapêutica para mastite 
Rev. Bras. Saúde Prod. Anim., Salvador, v.13, n.3, p. 701-711 jul./set., 2012 http://www.rbspa.ufba.br ISSN 15199940

estafilocócica nos rebanhos avaliados. Baixas taxas de resistência a drogas desses grupos foram relatadas por Bueno et al. (2003), Medeiros et al. (2009) e Zanette et al. (2010). Freitas et al. (2005), ao isolarem Staphylococcus coagulase positiva em rebanhos do Agreste pernambucano, e Ferreira et al. (2010), em isolados do Piauí, relataram taxas de 56 e $81,8 \%$ para a lincomicina, respectivamente. Uma vez que macrolideos e lincosamidas fazem parte de diversas formulações para uso intramamário, o conhecimento do perfil de sensibilidade de agentes causadores de mastite frente a essas drogas é importante para guiar a escolha da droga e evitar o surgimento de resistências.

Neste trabalho, foi selecionada a técnica da difusão do disco, mundialmente utilizada para avaliar susceptibilidade antimicrobiana de isolados animais e guiar a escolha do tratamento. Embora de fácil execução e baixo custo, apresenta algumas desvantagens como, por exemplo, a não detecção de mudanças sutis no perfil de isolados considerados sensíveis (KALMUS et al., 2011). Assim, as taxas de sensibilidade in vitro podem não refletir o real perfil do patógeno quando em contato com uma droga. Dessa forma, as taxas de resistência observadas podem ser bem mais elevadas do que o descrito.

Os resultados encontrados neste trabalho demonstram que $S$. aureus penicilina resistente está presente em rebanhos leiteiros do município de Garanhuns, o que sugere uso abusivo dessas drogas e, ao mesmo tempo, indica um risco potencial à saúde de consumidores, uma vez que o leite e derivados podem veicular resíduos de antibióticos e/ou cepas de $S$. aureus resistentes.

\section{AGRADECIMENTOS}

Os autores agradecem ao CNPq, pelo suporte financeiro (Processo $n^{\circ}$ 505912/2008-2), a UFRPE-UAG e Clínica de Bovinos de Garanhuns.

\section{REFERÊNCIAS}

\begin{abstract}
AMÁBILE-CUEVAS, C.F. Antibiotic resistance in Mexico: a brief overview of the current status and its causes. The journal of Infeccion in Developing
\end{abstract} Countries, v.4, n.3, p.126-131, 2010.

ANDRADE, M.A.; FILHO, F.C.D.; MESQUITA, A.J.; ROCHA, P.T. Sensibilidade in vitro de Staphylococcus aureus isolados de amostras de leite de vacas com mastite subclínica. Ciência Animal Brasileira, v.1, n.1, p.53-57, 2000.

ANON. Clinical Laboratory Standards Institute. Performance standards for antimicrobial disk and dilution susceptibility tests for bacteria isolated from animals. 3th ed. Wayne: CLSI, 2008. 116p.

BARKEMA, H.W.; SCHUKKEN, Y.H.; ZADOKS, R.N. The role of cow, pathogen, and treatment regimen in the therapeutic success of bovine Staphylococcus aureus mastitis.

Journal of Dairy Science, v.89, n.6, p.1877-1895, 2006.

BETANCOURT, O.; SCARPA, C.; VILLAGRÁN, K. Estudio de resistencia de cepas de Staphylococcus aureus aisladas de mastitis subclínica bovina frente a cinco antibióticos en tres sectores de la ix región de Chile.

Revista Científica, v.13, n.5, p.413417, 2003. 
Rev. Bras. Saúde Prod. Anim., Salvador, v.13, n.3, p. 701-711 jul./set., 2012 http://www.rbspa.ufba.br ISSN 15199940

BRITO, J.R.F.; BRITO, M.A.V.P. Programas de controle das mastites causadas por microrganismos contagiosos e do ambiente. Juiz de Fora: EMBRAPA-CNPGL, 1998. 25p. (Documentos, 71).

BUENO, V.F.F.; NICOLAU, E.S.; MESQUITA, A.J.; RIBEIRO, A.R.; SILVA, J.A.B.; COSTA, E.O.; COELHO, K.O.; COUTO, D.V. Etiologia e suscetibilidade à antimicrobianos dos agentes da mastite bovina isolados na região de Pirassununga - SP - Brasil. Revista de Patologia Tropical, v.32, n.1, p.33-44, 2003.

CORRÊA, I.; CORRÊA, M.G.P.; MARIN, J.M. Antimicrobial susceptibility of strains of coagulase positive Staphylococcus isolated from mastitis bovine milk. ARS Veterinária: Revista de Medicina Veterinária, v.21, n.1, p.69-76, 2005.

DOHOO, I.R.; SMITH, J.; ANDERSEN, S.; KELTON, D.F.; GODDEN, S. Diagnosing intramammary infections: Evaluation of definitions based on a single milk sample. Journal of Dairy Science, v.94, p.250-261, 2011.

FERREIRA, J.L.; PIGATTO, C.P.; LINS, J.L.F.H.A.; AGUIAR FILHO, J.L.C.; CAVALCANTE, T.V. Bactérias causadoras de mastite subclínica em rebanhos leiteiros no município de Teresina, Piauí. Revista Científica Eletrônica de Medicina Veterinária, v.8, n.14, 2010. Disponível em: < http://www.revista.inf.br/veterinaria14/a rtigos/RCEMV-AnoVIII-Edic14Art08.pdf>. Acesso em: 16 ago. 2011
FREITAS, M.F.L.; PINHEIRO JÚNIOR, J.W.; STAMFORD, T.L.M.; RABELO, S.S.A.; SILVA, D.R.; SILVEIRA FILHO, V.M.; SANTOS, F.G.B.; SENA, M.J.; MOTA, R.A. Perfil de sensibilidade antimicrobiana in vitro de Staphylococcus coagulase positivos isolados de leite de vacas com mastite no agreste do Estado de Pernambuco. Arquivos do Instituto Biológico, v.72, n.2, p.171-177, 2005.

HILLERTON, J.E.; KLIEM, K.E. Effective treatment of Streptococcus uberis clinical mastitis to minimize the use of antibiotics. Journal of Dairy Science, v.85, n.4, p.1009 - 1014, 2002.

KALMUS, P.; AASMAC, B.; KARSSIN, A.; ORRO, T.; KASK, K. Udder pathogens and their resistance to antimicrobial agents in dairy cows in Estonia. Acta Veterinaria Scandinavica, v.53, n.1, 2011.

KLOOS, WE. Systematic and the natural history of Staphylococci. 1. Journal of Applied Bacteriology, v.69, n.19, p.2537, 1990. Suppl.

LARANJA, L.F.; MACHADO, P.F. Ocorrência de mastite bovina em fazendas produtoras de leite $\mathrm{B}$ no estado de São Paulo. Revista Scientia Agricola, v.51, n.3, p.578-585, 1994.

\section{MAcFADDIN, J.F. Biochemical tests for identification of medical bacteria. $3^{\text {th }}$.ed. Washington: Williams \& Wilkins, 2000. 912p.}

MEDEIROS, E.S.; MOTA, A.M.; SANTOS, M.V.; FREITAS, M.F.L.; PINHEIRO JÚNIOR, J.W.; TELES, J.A.A. Perfil de sensibilidade microbiana in vitro de linhagens de Staphylococcus spp. isoladas de vacas com mastite subclínica. Pesquisa Veterinária Brasileira, v.29, n.7, p.569-574, 2009. 
Rev. Bras. Saúde Prod. Anim., Salvador, v.13, n.3, p. 701-711 jul./set., 2012 http://www.rbspa.ufba.br ISSN 15199940

MOREIRA, P.C.; SILVA, L.A.F.; MESQUITA, A.J. Resistência de Staphylococcus coagulase positiva e Streptococcus sp. isolados do leite de vacas com mastite clínica na bacia leiteira de Goiânia. Anais das Escolas de Agronomia e Veterinária, v.27, n.2, p.61-68, 1997.

MORONI, P.; PISONI, G.; ANTONINI, M.; VILLA, R.; BOETTCHER, P.; CARLI, S. Susceptibilidade às drogas antimicrobianas de Staphylococcus aureus oriundos de mastites bovinas subclínicas na Itália. Journal of Dairy Science, v.89, p.2973-2976, 2006.

NAM, H.; LEE, A.; JUNG, S.; KIM, G.; WEE, S.; LIM, S. Antimicrobial susceptibility of Staphylococcus aureus and characterization of methicilimresistant Staphylococcus aureus from bovine in Korea. Foodborne Pathogens and Disease, v.8, n.2, p.231-238, 2011.

NATIONAL MASTITIS COUNCIL NMC. Microbiological procedures for the diagnosis of bovine udder infeccion. 4.ed. Arlington, 2004. 46p.

PELLEGRINO, M.S.; FROLA, I.D.; ODIERNO, L.M.; BOGNI, C.I. Mastitis bovina: resistencia a antibióticos de cepas de Staphylococcus aureus aisladas de leche. Revista Eletrônica de Veterinária, v.12, n.7, 2011.Disponivel em:

<http://www.revista.org/revistas/redvet/n 070711.html>. Acesso em: 14 jun. 2011.

PERSSON, Y.; NYMAN, A.J.; GRÖNLUND-ANDERSSON, U. Etiology and antimicrobial susceptibility of udder pathogens from cases of subclinical mastitis in dairy cows in Sweden. Acta Veterinaria Scandinavica, v.53, n.1, p.36, 2011.
QUINN, P.J.; CARTER, M.E.; MARKEY B.; CARTER, G.R. Clinical veterinary microbiology. London: Mosby, 1994. 648p.

REINOSO, E.; BETTERA, S.; ODIERNO, L.; BOGNI, C. rep-PCR of Staphylococcus aureus trains isolated from bovine mastitis in Argentina. Brazilian Journal of Veterinary Research and animal Science, v.44, p.155-121, 2007.

SABOUR, P.M.; GILL, J.J.; LEPP, D.; PACAN, J.C.; AHMED, R.; DINGWELL, R.; LESLIE, K. Molecular typing and distribution of Staphylococcus aureus isolates in Eastern Canadian dairy herds. Journal of Clinical Microbiology, v.42, n.8, p.3449-3455, 2004.

SAHEBEKHTIARI, N.; NOCHI, Z.; ESLAMPOUR, M.A.; DABIRI, H.; BOLFION, M.; TAHERIKALANI, M.; KHORAMIAN, B.; ZALI, M.R.; EMANEINI, M. Characterization of Staphylococcus aureus strains isolated from raw milk of bovine subclinical mastitis in Tehran and Mashhad. Acta Microbiologica et Immunologica Hungarica, v.58, n.2, p.113-121, 2011.

SAS INSTITUTE. User's guide: statistics. Version 9.2. Cary. NC., 2008.

TAPONEN, S.; JANTUNEN, A.; PYÖRÄLÄ, E.; PYÖRÄLÄ, S.

Efficacy of targeted 5-day combined parenteral and intramammary tyreatment of clinical mastitis caused by penicillin-susceptible or penicillinresistant Staphylococcus aureus. Acta Veterinaria Scandinavica, v.44, n.1, p.53-62, 2003. 
Rev. Bras. Saúde Prod. Anim., Salvador, v.13, n.3, p. 701-711 jul./set., 2012 http://www.rbspa.ufba.br ISSN 15199940

ZAFALON, L.F.; LANGONI, H.; BENVENUTTO, F.; CASTELANI, L.; BROCCOLO, C.R. Aspectos epidemiológicos da mastite bovina causada por Staphylococcus aureus. Veterinária e Zootecnia, v.15, n.1, p.56-65, 2008.

ZANETTE, E.; SCAPIN, D.; ROSSI, E.M. Suscetibilidade antimicrobiana de Staphylococcus aureus isolados de amostras de leite de bovinos com suspeita de mastite. Unoesc \& Ciência - ACBS, v.1, n.1, p.65-70, 2010.
ZSCHÖCK, M.; EL-SAYED, A.; EISSA, N.; LÄMMLER, C.; CASTAÑEDA-VAZQUEZ. $\mathrm{H}$. Resistencia a penicilina $\mathrm{G}$ y oxacilina, de cepas de Staphylococcus aureus isoladas de mastitis bovina subclínica. Revista Veterinaria México, v.42, n.3, p.207-217, 2011.

Data de recebimento: 10/11/2011

Data de aprovação: 17/05/2012 